\title{
Multicenter Validation of the Vasoactive-Ventilation-Renal Score as a Predictor of Prolonged Mechanical Ventilation After Neonatal Cardiac Surgery
}

\author{
Short title: VVR Score after Neonatal Cardiac Surgery
}

Katherine Cashen, DOㄹ ; John M Costello MD, MPH2; Lisa M Grimaldi MD; Keshava Murty Narayana Gowda MD; ; Elizabeth AS Moser, MS; Kurt D Piggott MD ${ }^{6}$; Michael Wilhelm MD; Christopher W. Mastropietro MD ${ }^{8}$

\section{Affiliations: \\ ${ }^{1}$ Division of Critical Care, Department of Pediatrics, Children's Hospital of Michigan, Wayne State University, Detroit, MI \\ 2Division of Cardiology and Critical Care Medicine, Department of Pediatrics, Ann \& Robert H. Lurie Children's Hospital of Chicago, Northwestern University Feinberg School of Medicine, Chicago, IL \\ ${ }^{3}$ Division of Cardiovascular Intensive Care, Phoenix Children's Hospital, Department of Child Health, University of Arizona, College of Medicine, Phoenix, AZ \\ ${ }^{4}$ Division of Pediatric Critical Care Medicine, Department of Pediatrics, Cleveland Clinic, Cleveland, $\mathrm{OH}$ \\ ${ }^{5}$ Department of Biostatistics, Indiana University School of Medicine \& Richard M. Fairbanks School of Public Health, Indianapolis, IN \\ ${ }^{6}$ The Heart Center at Arnold Palmer Hospital for Children, Division of Pediatric Cardiac Intensive Care, Department of Pediatrics, University of Central Florida College of Medicine, Orlando, FL \\ ${ }^{7}$ Division of Pediatric Cardiac Intensive Care, Department of Pediatrics, University of Wisconsin, Madison, WI \\ ${ }^{8}$ Department of Pediatrics, Division of Critical Care, Indiana University School of Medicine, Riley Hospital for Children at Indiana University Health, Indianapolis, IN}

\section{Corresponding Author:}

Katherine Cashen DO

Division of Critical Care Medicine Children's Hospital of Michigan

3901 Beaubien, Detroit, MI 48201

Phone (313)745-5629

Fax (313) 966-0105

E-mail: kcashen@med.wayne.edu

Conflicts of Interest and Source of Funding: Ms. Moser's institution received funding from Riley Children's Hospital's Pediatric Critical Care Department in exchange for her biostatistical services to support their research. Dr. Mastropietro has received funding from Baez Law Firm for expert witness consultation. The remaining authors have disclosed that they do not have any potential conflicts of interest.

This is the author's manuscript of the article published in final edited form as:

Cashen, K., Costello, J., Grimaldi, L., Gowda, K. N., Moser, E. A., Piggott, K., ... Mastropietro, C. (2018). Multicenter Validation of the Vasoactive-Ventilation-Renal Score as a Predictor of Prolonged Mechanical Ventilation After Neonatal Cardiac Surgery*. Pediatric Critical Care Medicine, 19(11), 1015-1023. https://doi.org/10.1097/PCC.0000000000001694 


\section{ABSTRACT}

Objective: We sought to validate the vasoactive-ventilation-renal (VVR) score, a novel disease severity index, as a predictor of outcome in a multicenter cohort of neonates who underwent cardiac surgery.

Design: Retrospective chart review

Setting: Seven tertiary care referral centers

Patients: Neonates defined as age less than or equal to 30 days at the time of cardiac surgery Interventions: Ventilation index (VI), vasoactive-inotrope score (VIS), serum lactate, and VVR score were recorded for three postoperative time points: ICU admission, 6 hours and 12 hours. Peak values, defined as the highest of the three measurements, were also noted. VVR was calculated as follows: $\mathrm{VI}+\mathrm{VIS}+\Delta$ creatinine [change in creatinine from baseline*10]. Primary outcome was prolonged duration of mechanical ventilation, defined as greater than 96 hours. Receiver operative characteristic curves were generated and abilities of variables to correctly classify prolonged duration of mechanical ventilation were compared using area under the curve (AUC) values. Multivariable logistic regression modelling was also performed.

Measurements and Main Results: We reviewed 275 neonates. Median age at surgery was 7 days (25\%,75\%: 5,12), $86(31 \%)$ had single ventricle anatomy, and $183(67 \%)$ were classified as STAT mortality category 4 or 5 . Prolonged duration of mechanical ventilation occurred in 89 patients (32\%). At each postoperative time point, the AUC for prolonged duration of mechanical ventilation were significantly greater for the VVR score as compared to the VI, VIS, and serum lactate, with an AUC for peak VVR score of $0.82(95 \% \mathrm{Cl}: 0.77,0.88)$. On multivariable analysis, peak VVR score was independently associated with prolonged duration of mechanical ventilation, odds ratio (per 1-unit increase): 1.08 (95\% confidence interval: 1.04,1.12).

Conclusions: In this multicenter cohort of neonates who underwent cardiac surgery, the VVR score was a reliable predictor of postoperative outcome and outperformed more traditional measures of disease complexity and severity. 
Key Words: Congenital heart disease, Cardiac intensive care, postoperative care, mechanical ventilation, neonate

\section{Article Tweet}

In this multicenter study, we validated the VVR score as a reliable predictor of postoperative outcome in neonates who underwent cardiac surgery. 


\section{INTRODUCTION}

Neonates who undergo surgery for congenital heart disease are known to be at significant risk for postoperative morbidity and mortality (1-7). Although potential adverse outcomes have been well-described, the ability to identify patients at increased risk for poor outcomes has been challenging, in large part due to heterogeneity of the patients' anatomy and pathophysiology. Disease severity indices that quantify clinical data obtained within the early postoperative period and predict postoperative outcomes can have considerable utility and are sorely needed.

The vasoactive-ventilation-renal (VRR) score is a novel disease severity index that incorporates validated markers of cardiovascular, pulmonary, and renal function. In single center studies, it has been shown to be a robust predictor of outcomes in heterogenous populations of children recovering from surgery for congenital heart disease (8-10). These studies have included neonates (8-10), children with cyanotic congenital heart disease (9-10), children undergoing cardiopulmonary bypass (8-10), children not undergoing cardiopulmonary bypass (9-10), and adults with congenital heart disease (10). Most recently, the VVR score obtained at 12 hours postoperatively was shown to be a strong predictor of postoperative hospital length of stay and outperformed more traditional indices of disease complexity and illness severity including STAT mortality category, duration of cardiopulmonary bypass, vasoactive-inotrope score, ventilation index, and serum lactate (10).

For this study, we sought to perform the first multicenter validation of the VVR score utilizing a dataset containing neonates who underwent cardiac surgery at seven tertiary care centers within the United States (11). We hypothesized that the VVR would be a reliable predictor of outcome in this patient population and continue to outperform more traditional measures of disease complexity and severity. 


\section{METHODS}

\section{Design and Setting}

The study was a retrospective multicenter cohort study. We collected additional data on neonates who had previously been prospectively enrolled in an observational study examining extubation failure after neonatal cardiac surgery from January 1, 2015 through December 31, 2015 (11). Patients were enrolled from the following institutions: Riley Hospital for Children at Indiana University Health, Indianapolis, Indiana (coordinating center); Children's Hospital of Michigan, Detroit, Michigan; Cleveland Clinic, Cleveland, Ohio; Arnold Palmer Hospital for Children, Orlando, Florida; Phoenix Children's Hospital, Phoenix, Arizona; Ann \& Robert H. Lurie Children's Hospital of Chicago, Illinois; and American Family Children's Hospital, Madison, Wisconsin. The study was approved by the institutional review boards at all participating centers. Because of the observational nature of the data collected, the need for informed consent was waived.

\section{Study Population}

All neonates, defined as less than or equal to 30 days of age, who underwent pediatric cardiac surgery (with or without cardiopulmonary bypass) were reviewed. Neonates less than $2.5 \mathrm{~kg}$ who underwent ligation of the patent ductus arteriosus, patients with incomplete data for key exposure and outcome variables or patients placed on extracorporeal membrane oxygenation (ECMO) in the operating room prior to ICU arrival were excluded.

\section{Data Collection}

Data were collected via medical record review. Demographic and preoperative data collection included: age; sex; anthropometric data; estimated gestational age at birth; underlying cardiovascular diagnoses; presence of genetic anomalies; presence of non-cardiac anatomic anomalies; need for preoperative mechanical ventilation; and the occurrence of preoperative 
shock, infection, necrotizing enterocolitis, stroke or seizure. Perioperative data collected included: surgical procedure performed; Society of Thoracic Surgeons - European Association for Cardio-Thoracic Surgery Congenital Heart Surgery Mortality Categories (i.e., STAT Mortality Category) (4); duration of cardiopulmonary bypass and aortic cross clamping; and use of deep hypothermic arrest or antegrade cerebral perfusion. Postoperative data collected included: need for ECMO, inhaled nitric oxide therapy, CPR; presence of postoperative open sternotomy; or the occurrence of arrhythmias, acute kidney injury, infection, necrotizing enterocolitis, stroke, or seizure. We recorded mechanical ventilation parameters, arterial blood gas and lactate measurements, and doses of inotropic and vasopressor medications at three postoperative time points: ICU admission and 6 hours and 12 hours after ICU arrival. We also recorded preoperative serum creatinine and all serum creatinine measurements obtained within the first 12 postoperative hours.

\section{Derivation of the VVR}

The derivation of the VVR score has been described in detail elsewhere (8-10). In short, vasoactive inotrope score [VIS = dopamine dose $(\mathrm{mcg} / \mathrm{kg} / \mathrm{min})+$ dobutamine dose $(\mathrm{mcg} / \mathrm{kg} / \mathrm{min})$ +100 * epinephrine dose $(\mathrm{mcg} / \mathrm{kg} / \mathrm{min})+10$ * milrinone dose $(\mathrm{mcg} / \mathrm{kg} / \mathrm{min})+10,000$ * vasopressin dose $(\mathrm{U} / \mathrm{kg} / \mathrm{min})+100$ * norepinephrine dose $(\mathrm{mcg} / \mathrm{kg} / \mathrm{min})]$ and ventilation index $\left[\mathrm{VI}=(\text { Ventilator RR })^{*}(\mathrm{PIP}-\mathrm{PEEP})^{*} \mathrm{PaCO} 2 / 1000\right]$ were calculated at each postoperative time point.(12-15) VI and VIS were recorded as zero for patients not receiving mechanical ventilation or inotropic support, respectively. Baseline preoperative serum creatinine was subtracted from each postoperative serum creatinine measurement which we labeled $\Delta \mathrm{Cr}$. Admission values were typically utilized for admission and 6-hour VVR calculations and postoperative day 1 values were typically used for 12-hour values. For patients in which postoperative serum creatinine measurements were less than or equal to baseline, $\Delta \mathrm{Cr}=0$. VVR scores were calculated as follows: $\mathrm{VI}+\mathrm{VIS}+\Delta$ creatinine [change in serum creatinine from baseline ${ }^{*} 10$ ]. 


\section{Outcomes}

Our primary outcome variable was prolonged duration of mechanical ventilation, defined as greater than or equal to 96 hours based on previously published data of neonates recovering from cardiac surgery and ICD-10-CM procedure code 5A1955Z $(16,17)$. Measurements obtained after extubation from mechanical ventilation were not included in analyses focused on identifying predictor variables for prolonged mechanical ventilation. Secondary outcomes of interest were ICU length-of-stay (LOS), hospital LOS, and mortality. For secondary analyses, we defined prolonged duration of hospital LOS was defined as greater than 30 days or death prior to 30 days postoperatively.

\section{Statistical Analysis}

Descriptive statistics were used to characterize the patient population. Data are provided as median $(25 \%, 75 \%)$ for continuous variables and absolute counts with percentages for categorical variables. Bivariate comparisons of variables and outcomes in patients with and without prolonged duration of mechanical ventilation were performed using Wilcoxon rank-sum, chi-square, or Fisher exact tests as appropriate for individual variables. Receiver operating characteristic curves were generated and areas under the curve (AUC) with 95\% confidence intervals $(\mathrm{Cl})$ were calculated for prolonged duration of mechanical ventilation and VI, VIS, VVR, and serum lactate at all study time points, as well as for STAT mortality category and duration of cardiopulmonary bypass. Data for patients who were extubated prior to each postoperative time point were not included in the ROC analyses of data from time points following their liberation from mechanical ventilation. AUC values were compared using the method of DeLong, DeLong, and Clarke-Pearson as implemented by SAS.(18) 
For the multivariable regression analysis, variables with significance of 0.20 or less on bivariate comparison were considered for inclusion in the model. We also performed univariate and multivariable regression analyses to determine independent predictors of prolonged hospital LOS. Linearity in the logit was examined for continuous variables before model building; variables with evidence of nonlinearity were converted to categorical variables. To obtain the best model, stepwise selection was used with a significance level of 0.2 for entry into the model and 0.05 for staying in the model. The multivariable logistic model for our primary outcome variable, prolonged duration of mechanical ventilation, was also analyzed as a mixed model with a random effect for center. For these analyses, WVR scores were analyzed first as a continuous variable and secondly as a categorical variable using results of ROC characteristic results to identify appropriate cut points that maximize total accuracy and minimize weighted error ratios. The results of all multivariable analyses are provided as odds ratios (OR) with $95 \%$ confidence intervals $(\mathrm{Cl})$. All statistical analyses were performed with Stata version 14 (Stata Corp, College Station, Texas) and SAS version 9.4 (SAS Institute, Cary, North Carolina.)

\section{RESULTS}

We reviewed 293 patients, of which 16 were excluded due to ECMO initiation in the operating room and 2 were excluded due to incomplete data. The study population therefore consisted of 275 neonates, of which $173(63 \%)$ were male, $41(15 \%)$ were premature, and $43(16 \%)$ had a genetic anomaly. The median age at surgery was 7 days $(5,12)$ and median weight was $3.2 \mathrm{~kg}$ $(2.9,3.6)$. Eighty-six neonates $(31 \%)$ had single ventricle anatomy, $183(67 \%)$ were STAT mortality category 4 or 5 , and $71(26 \%)$ required preoperative mechanical ventilation. Deep hypothermic circulatory arrest or antegrade cerebral perfusion was employed in 107 (39\%) of patients and delayed sternal closure was used in 85 neonates (31\%), including 36 recovering from the Norwood operation. Additional data describing the study population is provided in Table 1. 
Prolonged duration of mechanical ventilation occurred in $89(32 \%)$ neonates. No patients died while receiving mechanical ventilation within 96 hours of surgery. Neonates with prolonged duration of mechanical ventilation were more likely to be premature, have non-cardiac anatomic anomalies, have received preoperative mechanical ventilation, have single ventricle anatomy, and be classified into a higher STAT Mortality Category (Table 1). Neonates with prolonged duration of mechanical ventilation were also more likely to have longer median cardiopulmonary bypass duration, received deep hypothermic circulatory arrest or antegrade cerebral perfusion, undergone delayed sternal closure, received inhaled nitric oxide, and experienced postoperative arrhythmias (Table 2).

Serum lactate, VIS, VI, and VVR measurements for all three postoperative time points and peak values are summarized in Table 3. Two patients were extubated in the operating room prior to ICU admission and were not included in these analyses. Four patients who were extubated between admission and 6-hours postoperatively and four patients who were extubated between 6 and 12 hours postoperatively were excluded from analyses of data obtained at 6-hours and 12-hours, respectively. The AUC values resulting from ROC characteristic analysis of each variable as predictors of prolonged duration of mechanical ventilation are also provided in Table 3. In this analysis, the VVR performed well with significantly greater AUC values than the corresponding VI, VIS and lactate. ROC curves for prolonged duration of mechanical ventilation and STAT Mortality Category, cardiopulmonary bypass duration, and peak VI, VIS, serum lactate, and VVR score are illustrated in Figure 1.

AUC values for VVR score at 6-hours and 12-hours postoperatively and peak VVR score were not statistically different, though all three AUC values were significantly greater than the AUC for VVR score at admission. For the multivariable analysis, we opted to examine peak VVR score 
which, unlike VVR score at 6 and 12 hours postoperatively, included measurements from all 273 patients who arrived to the ICU receiving mechanical ventilation (i.e., did not exclude any patient extubated between admission and 12 hours postoperatively). The results of the mixed multivariable regression analysis, which includes adjustments for random center effect, are shown in Table 4. Peak VVR score remained a strong independent predictor of prolonged duration of ventilation. Specifically, with each increase of 1 point in a patient's peak VVR score, the odds of prolonged duration of mechanical ventilation increased by $8 \%(\mathrm{OR}: 1.08 ; 95 \% \mathrm{Cl}$ 1.04-1.12). Additional variables that were independently associated with prolonged duration of mechanical ventilation were prematurity, preoperative mechanical ventilation, delayed sternal closure, postoperative inhaled nitric oxide use, and postoperative arrhythmia. ROC curves for the model and its individual components are shown in Figure 2. The AUC for the mixed model (which includes adjustment for possible center effect) is $0.92(95 \% \mathrm{Cl}: 0.89,0.96)$.

To simplify interpretation of the VVR score as a predictor of prolonged DMV, we repeated the multivariable analysis using peak VVR score as a categorical variable, with a cutoff value of 35 identified based on ROC characteristic analysis. For neonates with a peak VVR score greater than or equal to 35 , odds of prolonged duration of mechanical ventilation were more than three times that of patients with peak VVR score less than 35 (OR: 3.4; 95\% Cl 1.7-7.1). Further, patients with peak VVR greater than or equal to 35 had significantly longer postoperative ICU and hospital stay, and increased mortality (Table 5).

In the study population, 80 patients were considered to have prolonged postoperative hospital LOS: 78 patients required greater than 30 days of hospitalization after surgery and 2 patients died prior to 30 days postoperatively. Results of the multivariable analysis of this secondary outcome variable, unadjusted for center, are provided in Table 6. Peak VVR at 12 hours postoperatively was independently associated with prolonged LOS, along with genetic and non- 
cardiac abnormalities, single ventricle anatomy, preoperative mechanical ventilation, and the presence of clinically significant residual cardiac lesions. In a similar multivariable model that included the aforementioned variables but with peak VVR greater than or equal to 35 as a categorical variable, odds of prolonged LOS in neonates with a peak VVR score greater than or equal to 35 were more than three times that of the rest of the cohort (OR: $3.8 ; 95 \% \mathrm{Cl} 2.0-7.3$ ).

\section{DISCUSSION}

The VVR score was conceived as a multiorgan system severity of illness index for patients recovering from surgery for congenital or acquired heart disease that could improve upon traditional indices such as the VIS and serum lactate. While these latter measures have been demonstrated to be predictive of outcome in this patient population $(12-14,19)$, they are primarily reflective of postoperative cardiovascular health and do not quantify the contributions of other organ systems to postoperative disease acuity. Based on this concern, it was therefore hypothesized that a disease severity index containing measures of cardiovascular, respiratory, and renal function would be more reflective of the burden of illness being experienced by these fragile children. This hypothesis was proven correct in the several single center studies, in which the VVR was demonstrated to be a robust predictor of postoperative outcomes including prolonged duration of mechanical ventilation and postoperative length of stay.(8-10) Further, VVR measurements obtained at 6 and 12 hours postoperatively were identified as being more predictive of poor outcome than those obtained at admission, 24 and 48 hours.

After the initial single center work examining the VVR, this multicenter validation study was the logical next step. To accomplish this goal, we were able to utilize and add to data obtained in a previous prospective multicenter study of neonates who underwent cardiac surgery. From these data, we demonstrated that the peak VVR score obtained during the first 12 hours after admission was most predictive of prolonged duration of mechanical ventilation. Consistent with 
prior studies, the VVR score was superior to the VIS alone, ventilation index alone, serum lactate, STAT Mortality Category, and duration of cardiopulmonary bypass as a predictor of postoperative duration of mechanical ventilation and hospital length of stay. It is not surprising that the additional of postoperative ventilatory and to the VIS improved the ability to predict prolonged duration of mechanical ventilation. It was also not unexpected to find that quantification of postoperative disease severity is more predictive of postoperative outcome than pre- or intraoperative measures of disease complexity. The finding that the VVR score is more predictive of prolonged duration of mechanical ventilation than the ventilation index alone however is particularly notable, as it supports the notion that organ systems other than the respiratory system have a significant contribution to the postoperative ventilatory needs of this patient population. Despite our findings, we are not asserting that the VIS, ventilation index, or serum lactate have no value and should be abandoned in favor of the VVR, nor are we implying that our findings lessen the importance of STAT mortality category as the current standard for assessment of surgical complexity for children with congenital heart disease. On the contrary, we believe that the VVR should be added to this armamentarium of indices as a more comprehensive measure of postoperative disease severity.

In the most recent single center VVR study, a VVR score greater than 25 was identified as the best cutoff point distinguishing between children who were at risk for poor postoperative outcome and children who were not (10). Not surprisingly, in a cohort of patients that included only neonates, who tend to be more complex (i.e. $67 \%$ were classified as STAT Mortality Category 4 or 5), a peak VVR score greater than or equal to 35 was determined as the optimal cutoff value to identify patients most at risk for prolonged duration of mechanical ventilation. Moreover, peak VVR score greater than or equal to 35 within the first 12 hours of admission remained independently associated with prolonged duration of mechanical ventilation in a model that included several additional variables frequently associated with an extended postoperative 
course: prematurity, preoperative mechanical ventilation, delayed sternal closure, use of inhaled nitric oxide, and the occurrence of postoperative arrhythmias (20-26).

We opted to focus on the prolonged duration of mechanical ventilation as our measure of poor outcome in this study rather than prolonged length of stay or mortality for several reasons. First, there is a formal ICD-10 code for duration of mechanical ventilation greater than 96 hours, while no specific ICD-10 code or accepted definition exists for prolonged ICU or hospital stay. Interestingly, we found that $32 \%$ of patients within our cohort were classified by this ICD-10 code as having prolonged duration of mechanical ventilation, which is close to the upper $25 \%$ chosen in prior studies of the VVR (8-10). Our definition for prolonged duration of mechanical ventilation is further justified by data from a recent a large multicenter cohort of neonates recovering from cardiac surgery, which reported a median duration of mechanical ventilation of 70 hours (17). We were also aware that all but two of the patients were endotracheally intubated on mechanical ventilation upon arrival to the ICU. Indeed, prolonged duration of mechanical ventilation as an outcome measure would not have been ideal in a study in which a notable portion of patients were extubated in the operating room. Lastly, the low operative mortality rate in our study (4\%) precluded our ability to evaluate the VVR and other variables as predictors of this outcome. With lower operative mortality for congenital heart surgery in the current era, morbidity and resource consumption are receiving greater attention. We did however observe that postoperative ICU and hospital stay in patients with VVR score greater or equal to 35 were nearly twice that of patients with VVR score less than 35, while risk-adjusted operative mortality in patients with VVR score greater than or equal to 35 was nearly three times that of the rest of the cohort. A study with a greater absolute number of operative deaths would be required to further examine the ability of the VVR to predict mortality. 
The VVR score has many practical bedside applications. VVR scores obtained early in the postoperative course could allow clinicians to effectively quantitate disease severity and provide a reliable estimate of duration of mechanical ventilation and postoperative ICU and hospital stay to parents and staff. Likewise, these data could assist clinicians, nursing administrators, and respiratory therapists with resource allocation and quality improvement initiatives.

Academically, the WVR score has potential as a means of risk assessment, adjustment, and stratification in clinical research. On the other hand, though these data and earlier studies are promising, further validation in a multicenter data set that includes patients outside of the neonatal period should be conducted before widespread applications of the VVR score can be recommended.

This study contains the limitations inherent to a retrospective study design. Additionally, the VI, VIS, and VVR score cannot be reliably calculated for patients on ECMO, as these indices are not reflective of disease severity in this patient population. The use of EMCO however, in and of itself, is a significant risk factor for poor outcome in this patient population $(27,28)$. Further, because of the notable differences in outcomes in neonates and children who do and do not require ECMO after cardiac surgery, studies attempting to identify risk factors for poor outcomes in patients who require ECMO should consider focusing exclusively on this subgroup. It is also important to note that creatinine values within the neonatal population may be affected by maternal creatinine. Finally, vasoactive medication administration, ventilator support, and extubation readiness assessment were not protocolized, and as a result, were likely affected by variation in physician practice. Moreover, differences in intraoperative management may lead to differences postoperative vasoactive and ventilatory needs, as well as differences in early serum creatinine measurements after infant heart surgery (29). It is therefore encouraging that the VVR remained independently associated with prolonged duration of mechanical ventilation after adjustment for center effects. 


\section{CONCLUSION}

In a multicenter population of neonates undergoing surgery for congenital heart disease, the VVR calculated in the early postoperative recovery period was a reliable predictor of prolonged mechanical ventilation and outperformed more traditional measures of disease complexity and severity. Neonates with a peak VVR greater than or equal to 35 within the first 12 postoperative hours after cardiac surgery should be considered at increased risk for a more complicated postoperative course. 


\section{References}

1. Benavidez OJ1, Gauvreau K, Del Nido P, et al: Complications and risk factors for mortality during congenital heart surgery admissions. Ann Thorac Surg 2007;84:147-55.

2. Dorfman AT, Marino BS, Wernovsky G, et al: Critical heart disease in the neonate: Presentation and outcome at a tertiary care center. Pediatr Crit Care Med 2008; 9:193202.

3. Ohye RG, Sleeper LA, Mahony L, et al: Comparison of shunt types in the Norwood procedure for single-ventricle lesions. N Engl J Med 2010;362:1980-92.

4. Jacobs JP, O'Brien SM, Pasquali SK, et al: Variation in outcomes for risk-stratified pediatric cardiac surgical operations: an analysis of the STS Congenital Heart Surgery Database. Ann Thorac Surg 2012;94:564-71.

5. Feinstein JA, Benson DW, Dubin AM, et al: Hypoplastic left heart syndrome: current considerations and expectations. J Am Coll Cardiol 2012;59(1 Suppl):S1-4265.

6. Agarwal HS, Wolfram KB, Saville BR, et al: Postoperative complications and association with outcomes in pediatric cardiac surgery. J Thorac Cardiovasc Surg 2014;148:609-16.

7. Lex DJ, Tóth R, Czobor NR, et al: Fluid Overload Is Associated With Higher Mortality and Morbidity in Pediatric Patients Undergoing Cardiac Surgery. Pediatr Crit Care Med 2016;17:307-14.

8. Miletic KG, Spiering TJ, Delius RE, et al: Use of a novel vasoactive-ventilation-renal score to predict outcomes after paediatric cardiac surgery. Interact Cardiovasc Thorac Surg 2015;20:289-95

9. Miletic KG, Delius RE, Walters HL III, et al: Prospective validation of a novel vasoactiveventilation-renal score as a predictor of outcomes after pediatric cardiac surgery. Ann Thorac Surg 2016;101:1558-63 
10. Scherer B, Moser EAS, Brown JW, et al: Vasoactive-Ventilation-Renal Score Reliably Predicts Hospital Length-of-Stay after Surgery for Congenital Heart Disease. J Thorac Cardiovasc Surg 2016;152:1423-9

11. Mastropietro CW, Cashen K, Grimaldi LM, et al: Extubation Failure after Neonatal Cardiac Surgery. J Pediatr 2017;182:190-6

12. Gaies MG, Gurney JG, Yen AH, et al: Vasoactive-inotropic score as a predictor of morbidity and mortality in infants after cardiopulmonary bypass. Pediatr Crit Care Med 2010;11:234-8.

13. Davidson J, Tong S, Hancock H, et al: Prospective validation of the vasoactive-inotropic score and correlation to short-term outcomes in neonates and infants after cardiothoracic surgery. Intensive Care Med 2012;38:1184-90.

14. Gaies MG, Jeffries HE, Niebler RA, et al: Vasoactive-inotropic score is associated with outcome after infant cardiac surgery: an analysis from the Pediatric Cardiac Critical Care Consortium and Virtual PICU System Registries. Pediatr Crit Care Med 2014;15:529-37

15. Paret G, Ziv T, Barzilai A, Ben-Abraham R, et al: Ventilation index and outcome in children with acute respiratory distress syndrome. Pediatr Pulmonol 1998;26:125-8.

16. https://www.cms.gov/Medicare/Coding/lCD10/

17. Bennyworth BD, Mastropietro CW, Graham EM, et al: Variation in extubation failure rates after neonatal congenital heart surgery across Pediatric Cardiac Critical Care Consortium hospitals. J Thorac Cardiovasc Surg 2017;153 (6):1519-26.

18. DeLong ER, DeLong DM, Clarke-Pearson DL: Comparing the areas under two or more correlated receiver operating characteristic curves: a nonparametric approach. Biometrics 1988;44:837-45. 
19. Kalyanaraman M, DeCampli WM, Campbell AI, et al: Serial blood lactate levels as a predictor of mortality in children after cardiopulmonary bypass surgery. Pediatr Crit Care Med 2008;9:285-88.

20. Ip P, Chiu CS, Cheung YF: Risk factors prolonging ventilation in young children after cardiac surgery: Impact of noninfectious pulmonary complications. Pediatr Crit Care Med 2002;3:269-274.

21. Pagowska-Klimek I, Pychynska-Pokorska M, Krajewski W et al: Predictors of long intensive care unit stay following cardiac surgery in children. Eur J Cardiothorac Surg 2011;40:179-84.

22. Costello JM, Pasquali SK, Jacobs JP, et al: Gestational age at birth and outcomes after neonatal cardiac surgery: an analysis of the Society of Thoracic Surgeons Congenital Heart Surgery Database. Circulation 2014;129:2511-7.

23. Kalfa D, Krishnamurthy G, Duchon J, et al: Outcomes of cardiac surgery in patients weighing <2.5 kg: affect of patient-dependent and -independent variables. J Thorac Cardiovasc Surg 2014;148:2499-506.

24. Iliopoulos I, Burke R, Hannan R, et al: Preoperative Intubation and Lack of Enteral Nutrition are Associated with Prolonged Stay After Arterial Switch Operation. Pediatr Cardiol 2016;37:1078-84.

25. Erek E, Yalcinbas YK, Turkekul Y, et al: Indications and risks of delayed sternal closure after open heart surgery in neonates and early infants. World J Pediatr Congenit Heart Surg 2012;3:229-35.

26. Rekawek J, Kansy A, Miszczak-Knecht M et al: Risk factors for cardiac arrhythmias in children with congenital heart disease after surgical intervention in the early postoperative period. J Thorac Cardiovasc Surg 2007;133:900-4. 
27. Gupta P, Robertson MJ, Beam B, et al: Relationship of ECMO duration with outcomes after pediatric cardiac surgery: a multi-institutional analysis. Minerva Anestesiol 2015;81:619-27.

28. Merrill ED, Schoeneberg L, Sandesara P, et al: Outcomes after prolonged extracorporeal membrane oxygenation support in children with cardiac disease-Extracorporeal Life Support Organization registry study. J Thorac Cardiovasc Surg 2014;148:582-8.

29. Blinder JJ, Asaro LA, Wypij, D, et al: Acute kidney injury after pediatric cardiac surgery: a secondary analysis of the safe pediatric euglycemia after cardiac surgery trial. Pediatr Crit Care Med 2017;18:638-46. 
Table 1. Demographic, Anthropometric, and Preoperative Data

\begin{tabular}{|c|c|c|c|c|}
\hline Variable & $\begin{array}{l}\text { All patients } \\
(N=275)\end{array}$ & $\begin{array}{l}\text { DMV } \leq 96 h \\
(n=186)\end{array}$ & $\begin{array}{l}\text { DMV > 96h } \\
(n=89)\end{array}$ & $P$-value \\
\hline Age at surgery (days) & $7(5,12)$ & $7(5,13)$ & $7(5,11)$ & 0.62 \\
\hline Weight $(\mathrm{kg})$ & $3.2(2.9,3.6)$ & $3.3(3,3.7)$ & $3(2.6,3.4)$ & $<0.001$ \\
\hline Sex (male) & $173(63)$ & $119(64 \%)$ & $54(61 \%)$ & 0.60 \\
\hline Prematurity, $<37$ weeks & $41(15 \%)$ & $20(11 \%)$ & $21(24 \%)$ & 0.005 \\
\hline Genetic anomaly & $43(16 \%)$ & $24(13 \%)$ & $19(21 \%)$ & 0.07 \\
\hline Non-cardiac anatomic anomalies & $39(14 \%)$ & $20(11 \%)$ & $19(21 \%)$ & 0.02 \\
\hline Airway / respiratory anomaly & $15(5 \%)$ & $8(4 \%)$ & $7(8 \%)$ & 0.26 \\
\hline Neurologic anomaly or insult ${ }^{b}$ & $19(7 \%)$ & $11(6 \%)$ & $8(9 \%)$ & 0.35 \\
\hline Mechanical ventilation ${ }^{\mathrm{c}}$ & $71(26 \%)$ & $35(19 \%)$ & $36(40 \%)$ & $<0.001$ \\
\hline Shock & $39(14 \%)$ & $23(12 \%)$ & $16(18 \%)$ & 0.21 \\
\hline Infection & $15(5 \%)$ & $10(5 \%)$ & $5(6 \%)$ & 1.0 \\
\hline Necrotizing enterocolitis & $7(3 \%)$ & $4(2 \%)$ & $3(3 \%)$ & 0.69 \\
\hline Single ventricle anatomy & $86(31 \%)$ & $47(25 \%)$ & $39(44 \%)$ & 0.002 \\
\hline Hypoplastic left heart & $52(19 \%)$ & $21(11 \%)$ & $31(35 \%)$ & $<0.001$ \\
\hline Hypoplastic right heart & $24(9 \%)$ & $21(11 \%)$ & $3(3 \%)$ & 0.03 \\
\hline Heterotaxy & $10(4 \%)$ & $5(3 \%)$ & $5(6 \%)$ & 0.30 \\
\hline STAT Mortality Category & & & & $<0.001$ \\
\hline 1 & $5(2 \%)$ & $5(3 \%)$ & $0(0 \%)$ & $0 \%$ \\
\hline 2 & $42(15 \%)$ & $36(19 \%)$ & $6(7 \%)$ & $14 \%$ \\
\hline 3 & $45(16 \%)$ & $34(18 \%)$ & $11(12 \%)$ & $24 \%$ \\
\hline 4 & $136(49 \%)$ & $91(49 \%)$ & $45(51 \%)$ & $33 \%$ \\
\hline 5 & $47(17 \%)$ & $20(11 \%)$ & $27(30 \%)$ & $57 \%$ \\
\hline
\end{tabular}

Continuous variables represented as mean (standard deviation) or median $\left(25^{\text {th }} \%, 75^{\text {th }} \%\right)$; categorical data represented as absolute counts (\%)

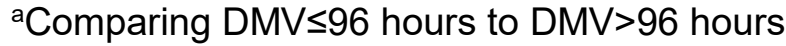

${ }^{b}$ Underlying neuroanatomic anomaly, or stroke or seizures prior to surgery

${ }^{c}$ Received mechanical ventilation until day of surgery

DMV: Duration of mechanical ventilation, STAT: Society of Thoracic Surgeons-European Association for Cardio-Thoracic Surgery Congenital Heart Surgery Mortality Category 
Table 2. Perioperative and ICU Admission Data

\begin{tabular}{lllll}
\hline Variable & $\begin{array}{l}\text { All patients } \\
(N=275)\end{array}$ & $\begin{array}{l}\text { DMV } \leq 96 h \\
(\mathrm{n}=186)\end{array}$ & $\begin{array}{l}\text { DMV }>96 h \\
(\mathrm{n}=89)\end{array}$ & $P$-value \\
\hline Cardiopulmonary bypass $(\min )$ & $129(0,184)$ & $119(0,162)$ & $150(84,217)$ & $<0.001$ \\
Aortic cross clamp $(\min )$ & $51(16,90)$ & $42(15,89)$ & $60(29,92)$ & 0.09 \\
DHCA & $67(24 \%)$ & $39(21 \%)$ & $28(31 \%)$ & 0.06 \\
ACP & $75(27 \%)$ & $41(22 \%)$ & $34(38 \%)$ & 0.005 \\
DHCA or ACP & $107(39 \%)$ & $62(33 \%)$ & $45(51 \%)$ & 0.006 \\
Delayed sternal closure & $85(31 \%)$ & $28(15 \%)$ & $57(64 \%)$ & $<0.001$ \\
Inhaled nitric oxide use & $58(21 \%)$ & $20(11 \%)$ & $38(43 \%)$ & $<0.001$ \\
Postoperative arrhythmias & $68(25 \%)$ & $32(17 \%)$ & $36(40 \%)$ & $<0.001$ \\
\hline
\end{tabular}

Continuous variables represented as mean (standard deviation) or median $\left(25^{\text {th }} \%, 75^{\text {th }} \%\right.$ ); categorical data represented as absolute counts (\%)

${ }^{a}$ Comparing DMV $\leq 96$ hours to DMV $>96$ hours

DMV: Duration of mechanical ventilation, ACP: antegrade cerebral perfusion; DHCA: Deep hypothermic circulatory arrest 
Table 3. ROC Analysis of Predictive Variables and Prolonged DMV

\begin{tabular}{|c|c|c|c|}
\hline Postoperative Variables & Median (IQR) & Maximum & AUC $(95 \% \mathrm{Cl})$ \\
\hline \multicolumn{4}{|l|}{ Admit: } \\
\hline Lactate (mg/dL) & $3(1.7-5)$ & 13.1 & $0.68(0.61-0.75)$ \\
\hline VIS & $8(5-11)$ & 30 & $0.69(0.62-0.76)$ \\
\hline VI & $19(15-25)$ & 112 & $0.72(0.66-0.79)$ \\
\hline VVR Score & $28(22-38)$ & 127 & $0.78(0.73-0.84)$ \\
\hline \multicolumn{4}{|l|}{ 6-hours: } \\
\hline Lactate $(\mathrm{mg} / \mathrm{dL})$ & $2.7(1.6-4.1)$ & 13.4 & $0.68(0.61-0.75)$ \\
\hline VIS & $8(5-11)$ & 37 & $0.68(0.61-0.75)$ \\
\hline $\mathrm{VI}$ & $17(13-22)$ & 96 & $0.76(0.70-0.82)$ \\
\hline VVR Score & $27(21-34)$ & 109 & $0.81(0.75-0.86)$ \\
\hline \multicolumn{4}{|l|}{ 12-hours: } \\
\hline Lactate (mg/dL) & $2.2(1.5-3.2)$ & 11.6 & $0.66(0.59-0.73)$ \\
\hline VIS & $8(5-12)$ & 64 & $0.69(0.62-0.75)$ \\
\hline VI & $16(12-21)$ & 74 & $0.74(0.67-0.80)$ \\
\hline VVR Score & $26(20-34)$ & 92 & $0.82(0.76-0.87)$ \\
\hline \multicolumn{4}{|l|}{ Peak in 12 hours: } \\
\hline Lactate $(\mathrm{mg} / \mathrm{dL})$ & $3.7(2.3-5.3)$ & 13.4 & $0.69(0.62-0.76)$ \\
\hline VIS & $10(6-13)$ & 64 & $0.70(0.63-0.77)$ \\
\hline VI & $21(16-27)$ & 112 & $0.78(0.71-0.84)$ \\
\hline VVR Score & $31(24-40)$ & 127 & $0.82(0.77-0.88)$ \\
\hline STAT Mortality Category & $4(3-4)$ & 5 & $0.66(0.60-0.72)$ \\
\hline CPB duration (min) & $129(0,184)$ & 413 & $0.64(0.57-0.71)$ \\
\hline
\end{tabular}

AUC: Area under the receiver operating characteristic curve; CPB: cardiopulmonary bypass; $\mathrm{Cl}$ : confidence interval; IQR: interquartile range; DMV: Duration of mechanical ventilation , STAT: Society of Thoracic Surgeons-European Association for Cardio-Thoracic Surgery Congenital Heart Surgery mortality category; VI: ventilation index; VIS: vasoactive inotrope score; VVR: vasoactive-ventilation-renal score 
Table 4. Multivariate Logistic Regression Analysis for Predictors of Prolonged Duration of Mechanical Ventilation (Mixed model adjusted for possible random effect of center)

\begin{tabular}{llc}
\hline Variable & Odds Ratio $(95 \%$ Confidence Interval $)$ & $P$-value \\
\hline Prematurity $(<37$ weeks $)$ & $3.5(1.3-9.1)$ & 0.012 \\
Preoperative ventilation & $5.3(2.2-12.7)$ & $<0.001$ \\
Delayed sternal closure & $10.8(4.2-28.2)$ & $<0.001$ \\
Inhaled nitric oxide use & $4.6(1.6-13.2)$ & 0.005 \\
Postoperative arrhythmias & $3.2(1.4-7.6)$ & 0.007 \\
Peak VVR in 1st 12 hours & $1.08(1.04-1.12)$ & $<0.001$ \\
\hline
\end{tabular}

VRR: vasoactive-ventilation-renal score 
Table 5. Clinical Outcomes

\begin{tabular}{llll}
\hline Outcome & $\begin{array}{l}\text { Peak VVR }<35 \\
(\mathrm{n}=169)\end{array}$ & $\begin{array}{l}\text { Peak VVR } \geq 35 \\
(\mathrm{n}=104)\end{array}$ & P-value \\
\hline Duration of ventilation (days) & $3(1,4)$ & $6(4,15)$ & $<0.001$ \\
ICU stay (days) & $8(4,15)$ & $14(9,32)$ & $<0.001$ \\
Hospital stay (days) & $14(9,25)$ & $29(18,50)$ & $<0.001$ \\
Mortality & $3(2 \%)$ & $9(9 \%)$ & 0.01 \\
\hline
\end{tabular}

Continuous variables represented as median $\left(25^{\text {th }} \%, 75^{\text {th }} \%\right)$; categorical data represented as absolute counts (percentages)

ICU: intensive care unit, VVR: vasoactive-ventilation-renal score 
Table 6. Multivariate Logistic Regression Analysis for Predictors of Prolonged Hospital Lengthof-Stay

\begin{tabular}{llc}
\hline Variable & Odds Ratio $(95 \%$ Confidence Interval) & $P$-value \\
\hline Genetic anomalies & $3.3(1.4-7.8)$ & 0.008 \\
Non-cardiac abnormalities & $5.0(2.1-12.1)$ & $<0.001$ \\
Preoperative ventilation & $3.9(1.9-8.0)$ & $<0.001$ \\
Single ventricle anatomy & $4.0(2.0-7.9)$ & $<0.001$ \\
Residual cardiac lesion & $4.8(2.1-10.9)$ & $<0.001$ \\
Peak VVR score in 1st 12 hours & $1.06(1.03-1.08)$ & $<0.001$ \\
\hline
\end{tabular}

VVR: vasoactive-ventilation-renal score 


\section{Figure Legends}

Figure 1: Receiver Operating Characteristic (ROC) curves for prolonged duration of mechanical ventilation. Exposure variables are STAT Mortality Category, duration of cardiopulmonary bypass (CPB), and peak serum lactate, vasoactive-inotrope score (VIS), ventilation index, and vasoactive-ventilation-renal (VVR) score at 12 hours postoperatively. The AUC was greatest for the VVR at 12 hours postoperatively, $\mathrm{P}<0.001$ for all comparisons.

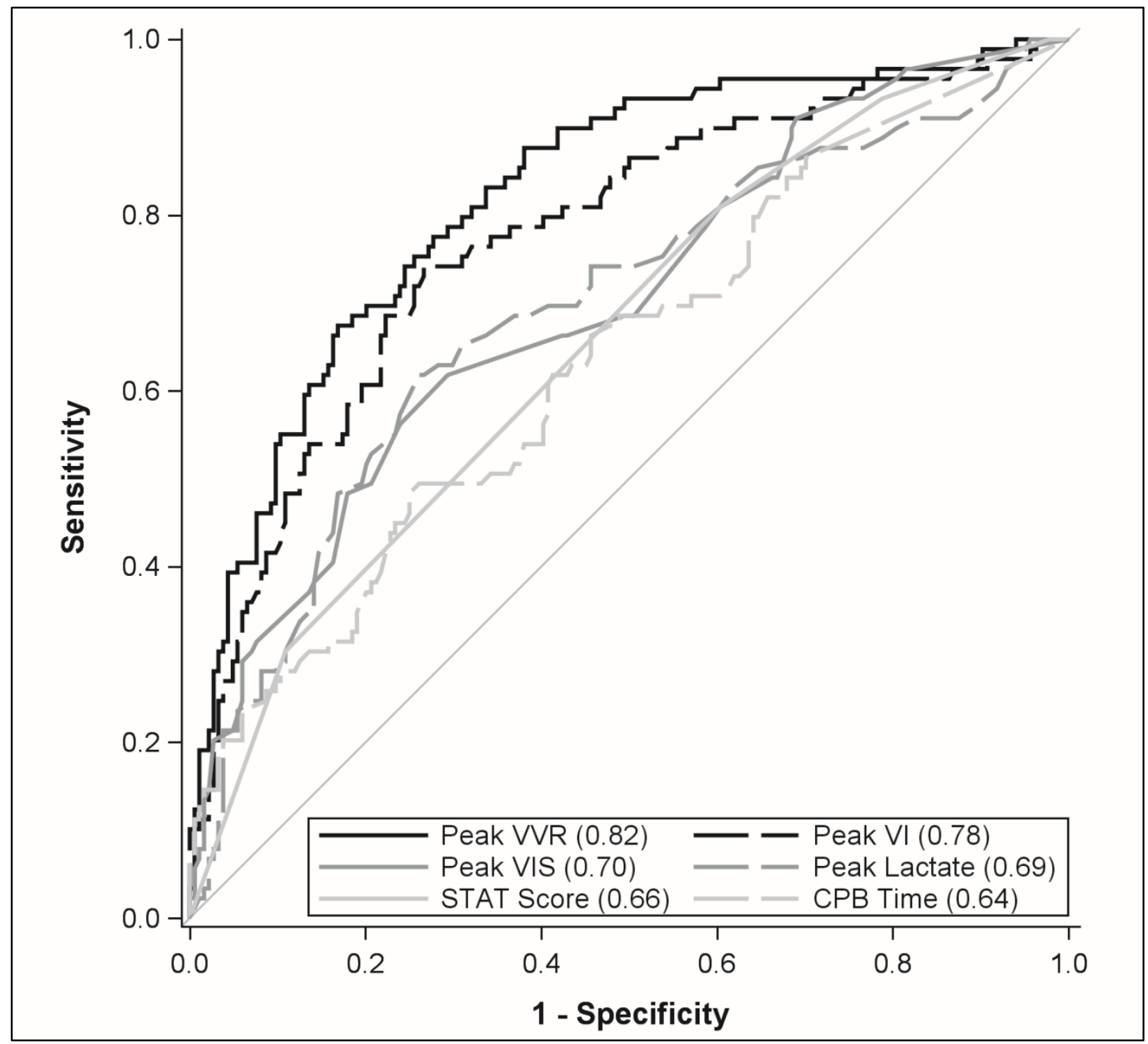


Figure 2: Receiver Operating Characteristic (ROC) curves for prolonged duration of mechanical ventilation. Depicted are the multivariable model and its individual components: vasoactiveventilation-renal (VVR) score at 12 hours postoperatively, delayed sternal closure, inhaled nitric oxide use, and postoperative arrhythmias, and prematurity. Area under the curve was 0.90 $(95 \% \mathrm{Cl}: 0.86,0.94)$ for the multivariable model and $0.92(95 \% \mathrm{Cl}: 0.89,0.96)$ for the mixed model (which included adjustment for center).

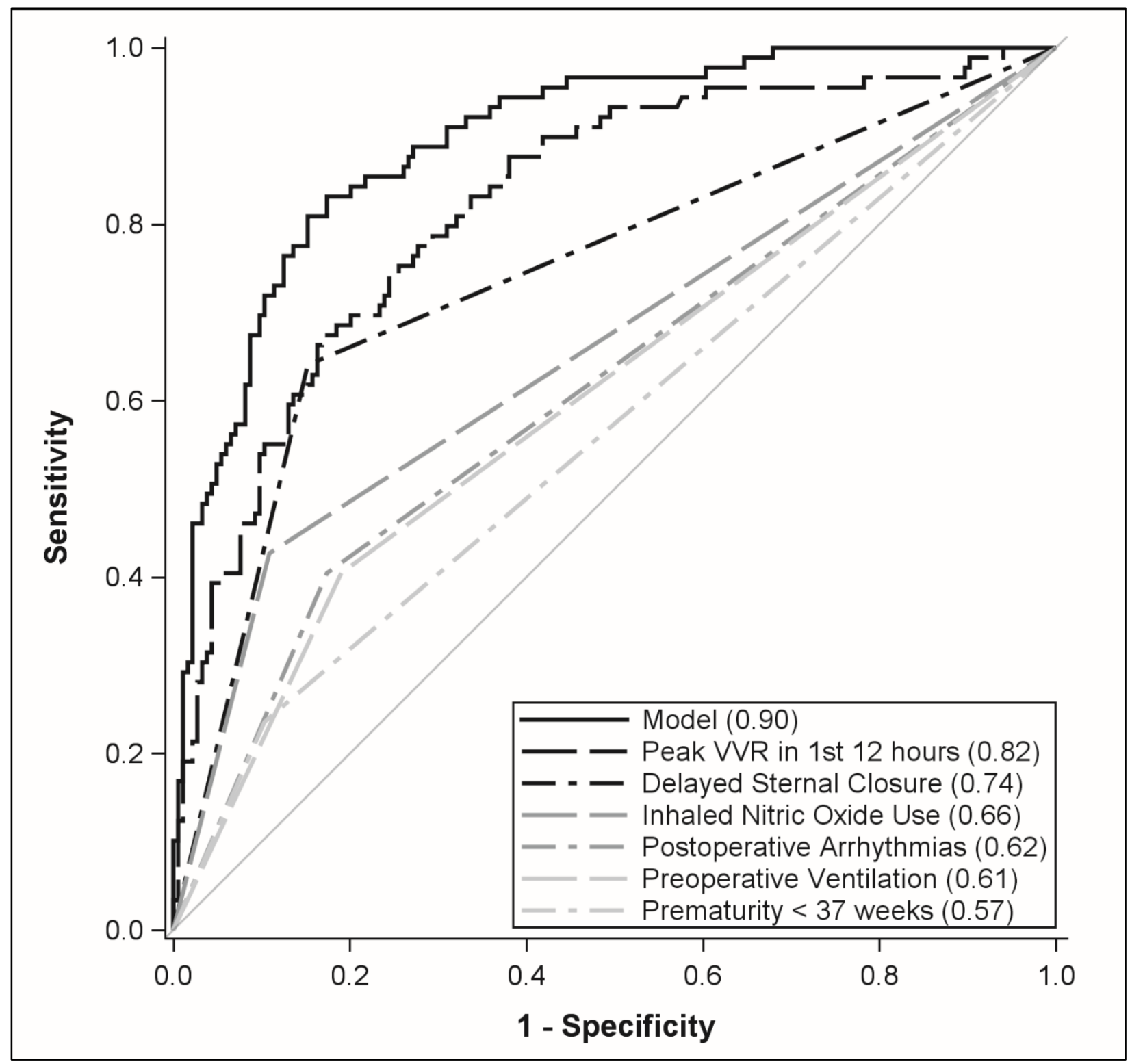

